\title{
Body Taping for Contour Surgery
}

\author{
Daniel Felix Kalbermatten · Reto Wettstein • \\ Paolo Erba · Ulrich Michael Rieger · Gerhard Pierer · \\ Wassim Raffoul
}

Received: 20 May 2008/Accepted: 7 October 2008/Published online: 22 November 2008

(C) Springer Science+Business Media, LLC and International Society of Aesthetic Plastic Surgery 2008

\begin{abstract}
Background Preoperative marking is of primary importance in body contouring and when precise simulation of skin excisions is difficult. Because the "cut as you go" principle can be delicate, especially in patients after massive weight loss, a simple and quick method is needed for preoperative planning. We suggest an approach that helps visualize the optimal skin incision lines and simulates the postoperative result by body taping.

Methods Twelve patients who underwent abdominal contouring, including classic and vertical abdominoplasties as well as dog ear and scar revision, were prospectively analyzed. The skin to be excised was preoperatively folded, taped, and then marked. The area marked was measured and compared with the actual intraoperatively resected area and the postoperative result was evaluated after 1 year by the patients and three surgeons.

Results With body taping, an $83 \%$ congruence between the preoperative planning and the surgery was obtained and

\section{F. Kalbermatten · P. Erba}

Department of Plastic, Reconstructive and Aesthetic Surgery,

University Hospital of Basel, 4031 Basel, Switzerland

D. F. Kalbermatten $(\bowtie) \cdot$ W. Raffoul

Department of Plastic, Reconstructive and Aesthetic Surgery, University of Lausanne, Rue de Bugnon 46, 1010 Lausanne,

Switzerland

e-mail: daniel.kalbermatten@bluewin.ch

\section{R. Wettstein}

Division of Plastic, Reconstructive and Aesthetic Surgery,

University Hospital of Geneva, 1211 Geneva, Switzerland

U. M. Rieger - G. Pierer

Klinik für Plastische und Wiederherstellungschirurgie A.ö. Landeskrankenhaus Innsbruck Medizinische, Universität Innsbruck, 6020 Innsbruck, Austria
\end{abstract}

only two patients had additional skin resected. No wound dehiscence and flap necrosis occurred and patients as well as surgeons scored the final body contour positively.

Conclusion Body taping is a simple, quick, and economic method for planning contour surgery with high accuracy as demonstrated by the low rate of intraoperative changes of the planned resection and low complication rate.

Keywords Skin resection - Tissue mobility .

Obesity surgery · Abdominoplasty · Post-bariatric surgery · Body contouring

Adhesive tapes represent a very conventional product in all areas of life, including surgery. Previously, adhesive tape was only rarely used for surgical planning [1, 2], and the use of tape to plan and simulate body contouring operations has not been evaluated yet. In contrast to the modern tools used to plan and analyze abdominal shape [3], taping is a simple and quick method that might be helpful for both the surgeon and the patient all the more in view of an increasing number of post-bariatric body-contouring procedures [4]. Therefore, we used taping to simulate extension and location of skin resection in different abdominal contour surgeries and compared the preoperative marking with the actual intraoperative resection.

\section{Patients and Methods}

A prospective clinical study was performed to evaluate the applicability of tapes for planning and marking in body contouring. Included were patients scheduled for surgery of the abdomen from January 2006 to June 2006. Surgical 
indications included excess skin after weight loss, scar revisions, and secondary contour corrections after abdominoplasty. The study followed the ethical guidelines of the local Academy of Medical Sciences and involved informed consent.

\section{Body Taping}

Preoperatively all patients were evaluated in the standing position. After palpation of the tissue excess, the skin was folded and maintained in the desired position with tape to simulate the result to be obtained (Durapore ${ }^{\mathrm{TM}}$ Surgical Tape, 3 M, St. Paul, MN, USA). Then the areas to be resected were marked with a pen (Edding 3000 permanent marker, Ahrensburg, Germany) (Figs. 1, 2, 3).

\section{Analyzed Parameters}

Age, gender, etiology of skin excess, and comorbidities were assessed. The surface of the marked resection area was measured and archived photographically. Intraoperatively, the actual resected area was assessed and compared to the preoperative marking. Patients were monitored for wound-healing complications and partial flap necrosis. One
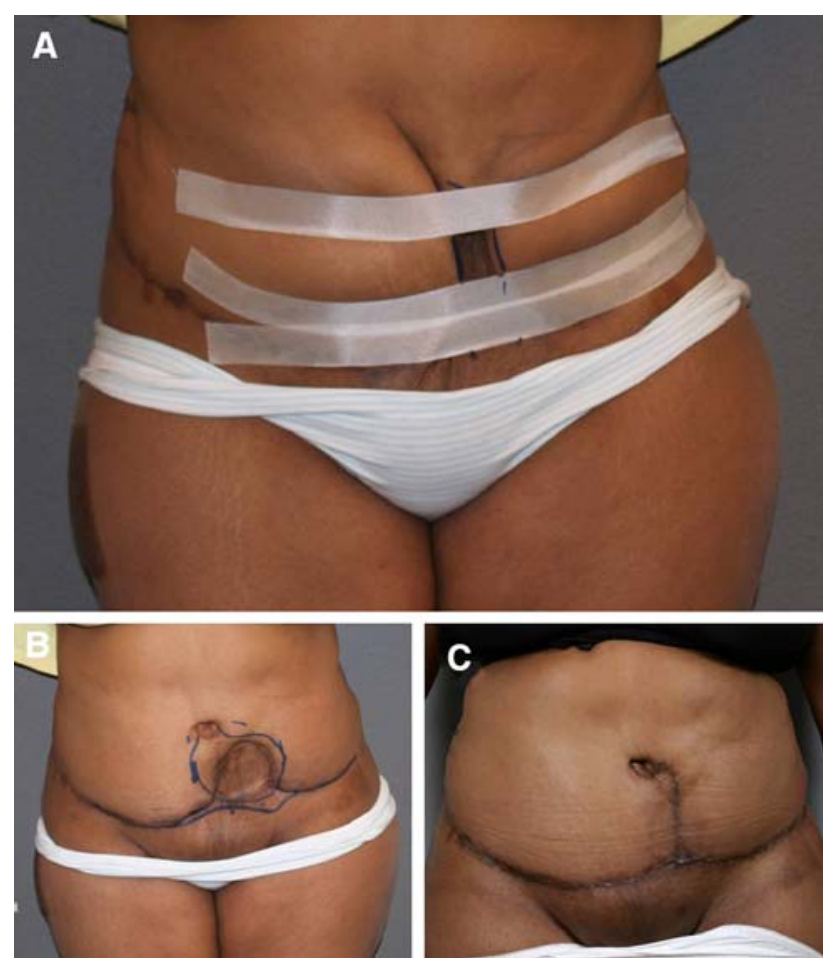

Fig. 1 Patient that presented to our clinic after abdominoplasty elsewhere with a extended skin flap necrosis that was initially debrided and covered with a split thickness skin graft. a Abdominal scar taped with three bands. b Markings of resection after release of tapes. c One-year postoperative result of abdominal scar correction using body taping
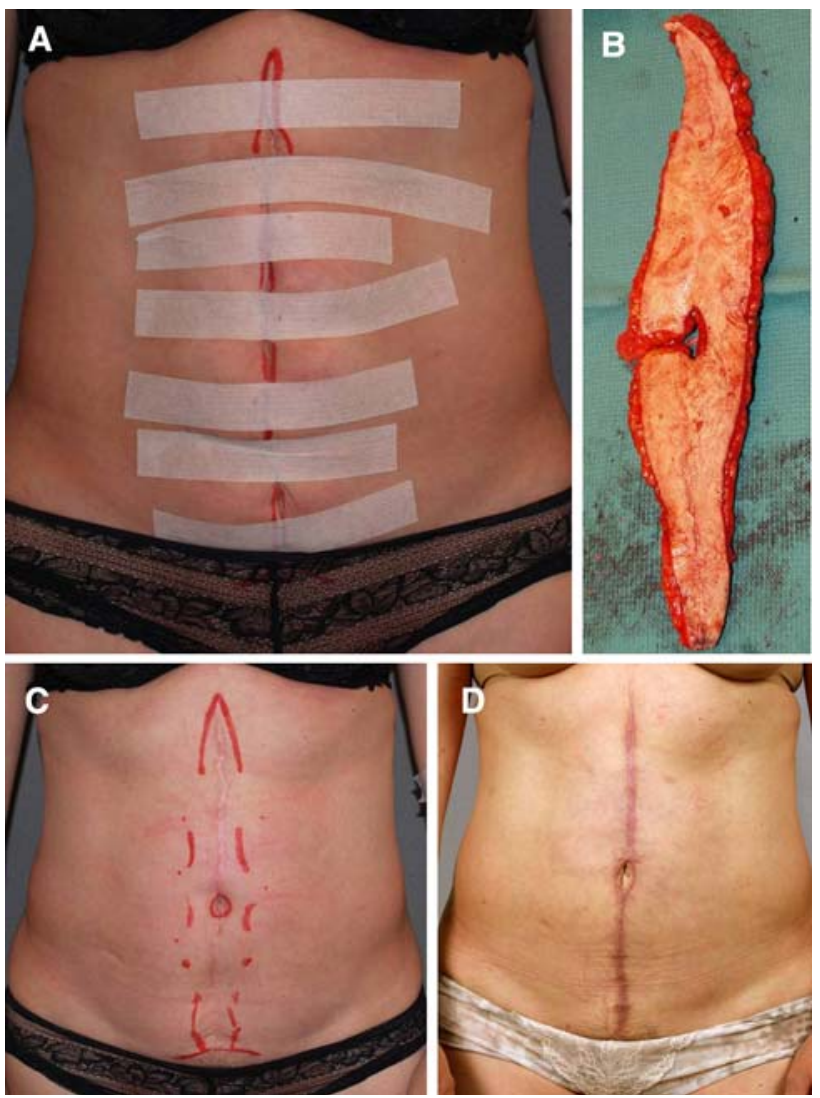

Fig. 2 Patient presenting after median laparotomy for contour optimization and scar revision. a Simulated vertical abdominoplasty. b Resected skin. c Markings after removal of tapes. d One-year postoperative result

year postoperatively, the result of the silhouette was evaluated by three surgeons and by the patient using a visual analog scale (VAS) ranging from 1 (optimal result) to 10.

\section{Results}

Twelve patients ( 3 male, 9 female, age $=38 \pm 6$ years) were included in the study. All patients gave their informed consent. Three patients suffered from dog ears after previous abdominoplasty, three were scheduled for vertical abdominoplasty after a previous median laparotomy, four were primary abdominoplasty patients, and two desired an abdominal scar correction. All patients had a history of weight loss secondary to diet (7 patients) or gastric bypass (5 patients). Comorbitities and risk factors were diabetes mellitus type II in two patients, arterial hypertension in two patients, and smoking by four patients.

Three to twelve strips of tape were used to preoperatively plan and mark the resection surfaces. The average resected area was $925 \mathrm{~cm}^{2}$ (range $=330-2014 \mathrm{~cm}^{2}$ ). Intraoperative resection corresponded to the preoperative taped area in 10 of the 12 cases. In two cases the skin 

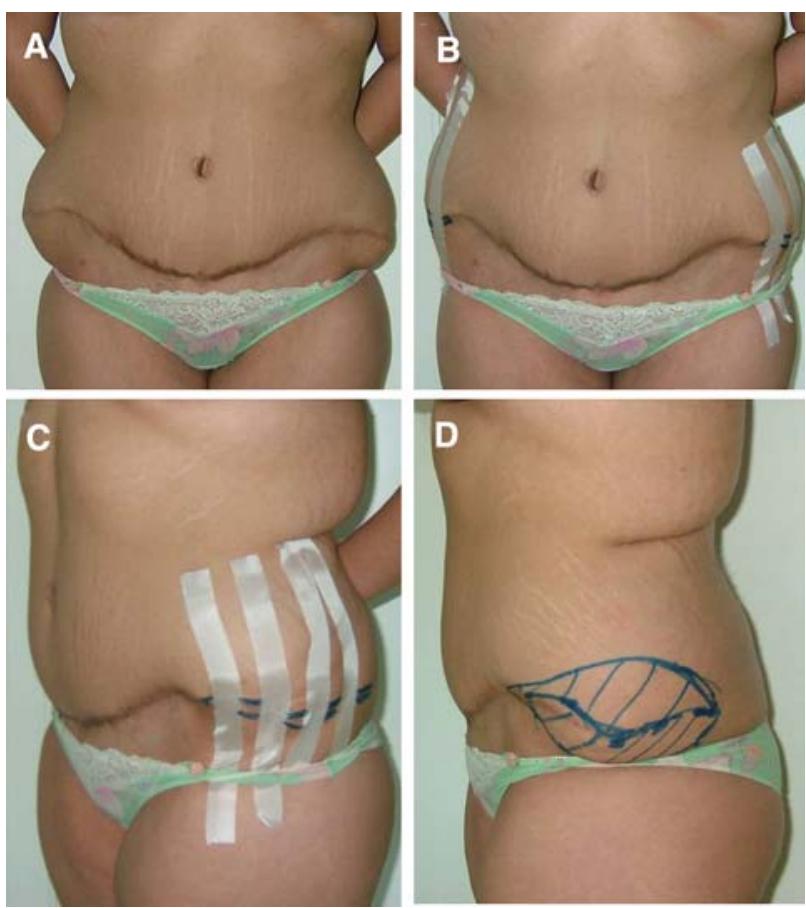

Fig. 3 a Patient with persistent tissue excess bilaterally after abdominoplasty. b Taped lateral skin excess for planning of resection lines. c Lateral view of the four bands of tape. d Markings after release of tapes shows resection lines laterally

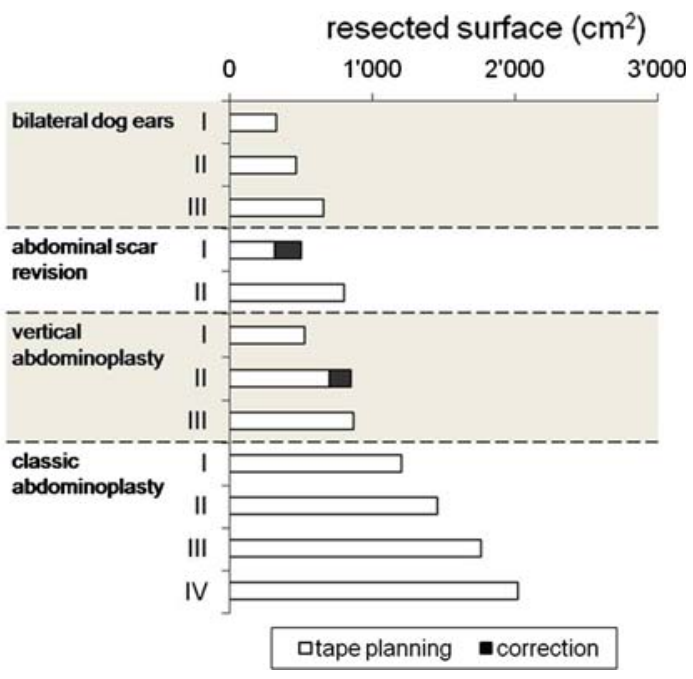

Fig. 4 Planned and actually resected surface areas. A high congruence of the planned and surgically resected areas was obtained and in only two cases was a correction of the planning necessary (black section of bars)

resection was increased intraoperatively. This resulted in a mean deviation from the preoperative planning of $27 \mathrm{~cm}^{2}$ (range $=0-180 \mathrm{~cm}^{2}$ ) (Fig. 4).

Postoperative controls revealed uneventful healing in all patients. At the 1-year follow-up control, high patient satisfaction was reflected in a VAS score of $3.3 \pm 1.2$ which was confirmed by evaluation by three surgeons whose VAS was $2.6 \pm 0.5$.

\section{Discussion}

Preoperative planning and marking is an integral part of any body-contouring operation [5, 6]. Ideally, techniques that are easily applicable and reliable support this essential step. Our experience with body taping in planning and simulating the extent of skin resection fulfills these criteria; only minimal time is required to do the taping and a $100 \%$ congruence was obtained in 10 of our 12 patients $(83 \%)$, i.e., the planned area was equal to the actual resected skin. Obviously, the amount of tape used is not cost-relevant.

This small series shows that the method works for different abdominal contouring indications and that in only two cases a minimal correction with extension of the excision was necessary. In addition, no wound-healing complications or necrosis occurred in this series which included patients with diabetes and tobacco abuse, and the satisfaction with the obtained silhouette was high. This implies that the planning with body taping was not only rapid and easy but accurate. We feel that this simple technique will be most helpful for patients presenting after massive weight loss with minimal subcutaneous fat and considerable skin laxity, not only in the trunk area where supraumbilical midline skin adhesion might limit the value of body taping, but also for brachioplasty and thigh lifts.

In conclusion, this small series shows the benefit of body taping, a simple and quick aid that can be used by every surgeon to make his operative planning easier without augmenting the costs or time for surgeon or patient.

\section{References}

1. Saxby PJ (1988) Inelastic tape to treat problems of tissue expansion. Br J Plast Surg 41(6):666-667

2. Botti G (1989) The use of adhesive tape to inform the patient preoperatively. Plast Reconstr Surg 83(3):571

3. Rieger U, Erba P, Wettstein R, Schumacher R, SchwenzerZimmerer K, Haug M, Pierer G, Kalbermatten DF (2008) Does abdominoplasty with liposuction of the love handles yield a shorter scar? an analysis with abdominal $3 d$ laser scanning. Ann Plast Surg 61(4):359-361

4. Chandawarkar RY (2006) Body contouring following massive weight loss resulting from bariatric surgery. Adv Psychosom Med 27:61-72

5. Wilkinson TS, Paletta FX (1969) Mesh tape for skin graft immobilization. Plast Reconstr Surg 44(1):31-36

6. Gibson EW, Poate WJ (1964) The use of adhesive surgical tape in plastic surgery. Br J Plast Surg 17:265-270 